\title{
Protective effects of glucocorticoid on liver injury in a rat sepsis model
}

\author{
XIAOLI LI* , MEIFENG LI* , LUYI LIU, XINGHAN TIAN and YAFENG LIANG \\ Department of Critical Care Medicine, The Affiliated Yantai Yuhuangding Hospital of Qingdao University, \\ Yantai, Shandong 264000, P.R. China
}

Received August 23, 2018; Accepted July 12, 2019

DOI: $10.3892 /$ etm.2019.7899

\begin{abstract}
Sepsis is one of the leading causes of death with unsatisfactory current treatments. The present study assessed the liver protective effect of glucocorticoids on different levels of inflammation in septic shock rats. A rat septic shock model was established by lipopolysaccharide (LPS) injection. Rats were divided into control (Control), high-inflammation treated with hydrocortisone (HT), high-inflammation non-treatment (HNT), low-inflammation treated with hydrocortisone (LT) and low-inflammation non-treatment (LNT) groups according to the levels of serum C-reactive protein (CRP), interleukin (IL)-6 and interferon (IFN) $-\gamma$. The mean arterial pressure and heart rate changes were continuously monitored and aspartate aminotransferase (AST) and alanine aminotransferase (ALT) levels were measured by an automatic biochemical analyzer. Hematoxylin and eosin (H\&E) staining was performed to observe the pathological changes of liver tissue. Western blot analysis was used to detect the expression of p38 mitogen-activated protein kinase (MAPK) and $\mathrm{NF}-\kappa \mathrm{B}$ protein. The results demonstrated that following 7 days of treatment, there were no obvious differences in the serum CRP, IL-6 and IFN- $\gamma$ levels between the HT group and the control group, whilst the HNT group, LT group and LNT group were significantly different compared with the HT and control groups. H\&E staining demonstrated that the liver cells in the HT group were homogeneous following 7 days of treatment. Western blot analysis determined that the phosphorylation levels of p38MAPK and $\mathrm{NF}-\kappa \mathrm{B}$ in HT group were reduced significantly compared with the LT group, while there was no obvious difference with the control group after 7 days
\end{abstract}

Correspondence to: Dr Yafeng Liang, Department of Critical Care Medicine, The Affiliated Yantai Yuhuangding Hospital of Qingdao University, 20 Yuhungding East Road, Zhifu, Yantai, Shandong 264000, P.R. China

E-mail: lixiaoli0799@yeah.net

*Contributed equally

Key words: glucocorticoids, liver injury, septic shock,

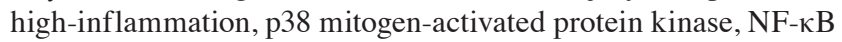

treatment. The present results indicated that glucocorticoids have better therapeutic effect on septic shock rats with high-inflammation compared with low-inflammation rats. The present study provides a novel approach for glucocorticoid treatment of septic shock.

\section{Introduction}

Septic shock is one of the leading causes of death in critical care medicine with an incidence that increases year by year (1). Although technology for the treatment of septic shock continues to advance, disease mortality rate remains high (2). The pathogenesis of sepsis is extremely complex, involving many systems such as immunity, neuroendocrine and coagulation systems (3-5). Inflammatory reactions and immune dysfunction are important parameters in patients with septic shock. A previous study determined that septic shock occurs as a result of excessive deterioration of the systemic inflammatory response with the dynamic balance of pro-inflammatory and anti-inflammatory mediators determining the occurrence and development of septic shock (6). The excessive uncontrolled activation of inflammatory factors and disturbances in immune function can lead to multiple organ failure and death.

Interleukin (IL)-6, a pro-inflammatory mediator, is an important stimulator of acute protein synthesis by the liver during sepsis (7). It has a long metabolism time and is released in large quantities by stimulated endothelial cells and macrophages. IL-6 is considered an important marker of sepsis (8). C-reactive protein (CRP) is a non-specific systemic inflammatory marker protein (9), which is a typical acute-phase reaction protein produced and secreted by hepatocytes (10). The level of CRP is associated with the inflammatory response and repair level of the body, when inflammation occurs, CRP level rises rapidly in a short time, with the recovery and remission of the disease, CRP can quickly return to normal level (11). Interferon- $\gamma($ IFN- $\gamma)$ is a type of interferon that regulates immune function, resists viruses, prevents tumorigenesis and inhibits the secretion of cells (12-14).

Glucocorticoids are an important class of regulatory molecules in the body (15). They have important regulatory roles in development, growth, metabolism and immune function (16-18). In clinical practice, they are widely used as effective anti-inflammatory and immunosuppressive agents (19). However, possible adverse reactions include 
secondary infections, gastrointestinal bleeding, and hyperglycemia (20). Therefore, further research into glucocorticoids is required. In the present study, hydrocortisone was administered to high-inflammation and low-inflammation rats to assess its treatment effect and its protective effect on the liver. This may provide a theoretical basis for the scientific and rational application of glucocorticoids for the treatment of septic shock.

\section{Materials and methods}

Establishment of septic shock rat model. A total of 60 Sprague-Dawley rats (male; age, 6-8 weeks; weight, 190 \pm 10 g). were purchased from Beijing Weitong Lihua Experimental Animal Technology Co., Ltd. Rats were housed in SPF animal room at $20-26^{\circ} \mathrm{C}$, relative humidity was $50-60 \%$ and $12-\mathrm{h}$ light/dark cycle. Rats have free access to food and drink.

Rats were randomly divided into control $(n=12)$ and model groups $(n=48)$. They were fasted overnight prior to surgery with free access to drinking water. Rats were anesthetized with an intraperitoneal injection of $1 \%$ pentobarbital sodium (40 mg/kg; Jiangsu Hengrui Medicine Co., Ltd.). Following supine fixation, an incision was made in the right groin to separate the femoral artery. The end of the heart was ligated with silk thread (size 0) and a heparinized $22 \mathrm{G}$ cannula was inserted proximally. The silk thread was ligated and fixed. The other end of the cannula was connected to a blood pressure monitor and the mean arterial pressure (MAP) was measured. The left external jugular vein was isolated and placed in the tube as an injection drug route. Following completion of the above surgery, animals were stabilized for $10 \mathrm{~min}$ prior to drug injection.

Model group rats were injected with lipopolysaccharide (10 mg/kg; Sigma-Aldrich; Merck KGaA) for $10 \mathrm{~min}$. The control group was injected with an equal volume of normal saline calculated from rat body weight (average $0.2 \mathrm{ml}$ ). The vital signs monitor (Indus Instruments) continuously monitored changed in MAP and heart rate. Rats were observed for the presence or absence of weakness, chills, bloating, diarrhea and erect hairs. Establishment of a successful shock model was considered when MAP declined by at least $50 \%$ and rats were observed for the presence of weakness, chills, bloating, diarrhea and erect hairs. Following successful modeling, fluid resuscitation commenced. The resuscitation fluid was treated with a compound sodium chloride injection $(30 \mathrm{ml} / \mathrm{kg} / \mathrm{h})$ and femoral vein quick rehydration was used to restore normal blood pressure. A muscular injection of penicillin (100,000 U/Kg) (North China Pharmaceutical Company, Ltd.) was then administered. All experiments were performed in accordance with the principles established by the Affiliated Yantai Yuhuangding Hospital of Qingdao University Animal Ethics Committee (project no. YHDQD1632; ethical approval date, 15/03/2017).

Experimental groups. Following resuscitation and penicillin treatment, rats were anesthetized with an intraperitoneal injection of $1 \%$ pentobarbital sodium $(40 \mathrm{mg} / \mathrm{kg}$ ), following which $0.5 \mathrm{ml}$ carotid artery blood was collected and serum was obtained via centrifugation $(1,000 \times \mathrm{g}$ for $5 \mathrm{~min}$, at room temperature). IL-6 (cat. no. SEKR-0005-96T; Solarbio Science \& Technology Co., Ltd.), CRP (cat. no. ABIN368062; antibodies-online) and IFN- $\gamma$ (cat. no. DY585; R\&D Systems, Inc.) content in the serum of rats were detected via ELISA. Following resuscitation and penicillin treatment, prior to glucocorticoid treatment and according to the levels of IL-6, CRP and IFN- $\gamma$, rats were equally divided into a high-inflammation group (CRP $\geq 500 \mathrm{ng} / \mathrm{ml}$; IL-6 $\geq 50 \mathrm{pg} / \mathrm{ml}$; IFN- $\gamma \geq 150 \mathrm{pg} / \mathrm{ml} ; \mathrm{n}=24$ ) and the content of IL-6, CRP and IFN- $\gamma$ below the previous parameters were low-inflammation group $(n=24)$. The high and low-inflammation groups were then equally divided into a glucocorticoid therapy group $(n=12)$ and a non-glucocorticoid therapy group $(n=12)$, and intervention therapy was also performed. The glucocorticoid-treated group was injected with hydrocortisone $(6 \mathrm{mg} / \mathrm{kg}$; Sigma-Aldrich; Merck KGaA) in the external jugular vein and the non-glucocorticoid-treated group was injected with the same amount of saline. The treatment was administered $6 \mathrm{~h}$ the rats resuscitation, once a day for 7 days.

Monitoring of arterial pressure and heart rate. The dietary activities (eat and drink) of rats were observed in each group. The vital sign detector (Indus Instruments) was used to continuously monitor the arterial pressure and heart rate changes in each group of rats during modeling and treatment for 7 days.

Liver function test. According to the manufacturer's instructions (Beijing Solarbio Science \& Technology Co., Ltd.), the contents of serum alanine aminotransferase (ALT; cat. no. BC1555) and aspartate aminotransferase (AST; cat. no. BC1565) were measured using an automatic biochemical analyzer (Beckman Coulter, Inc.).

ELISA detection of serum IL-6, CRP and IFN- $\gamma$ content. Arterial blood was collected from each group of rats and the serum was separated via centrifugation at $1,000 \mathrm{x} g$ for $10 \mathrm{~min}$, at room temperature. ELISA kits were used to detect the changes of serum IL- $6, \mathrm{CRP}$ and IFN- $\gamma$ contents according to the manufacturer's protocol.

Hematoxylin and eosin $(H \& E)$ staining. Liver tissue was removed, fixed in $4 \%$ paraformaldehyde at $4^{\circ} \mathrm{C}$ for $12 \mathrm{~h}$, embedded in paraffin and cut into $4 \mu \mathrm{m}$ sections. Samples were rinsed twice with xylene for $10 \mathrm{~min}$ and dehydrated with a descending alcohol series (100\% alcohol for $5 \mathrm{~min} ; 95 \%$ alcohol for $5 \mathrm{~min}$; $75 \%$ alcohol for $5 \mathrm{~min}$ ). Tissue was then stained with hematoxylin for $10 \mathrm{~min}$ and eosin for $5 \mathrm{~min}$ at room temperature (Beijing Solarbio Science \& Technology Co., Ltd.). Samples were washed with $100 \%$ alcohol and rinsed twice with xylene for 5 min each. Neutral gum was used for sealing. Light microscopy (Olympus Corporation; magnification, $\mathrm{x} 400$ ) was used to observe the pathological features of liver tissue.

Western blot analysis. Liver tissue was extracted using protein extraction reagents (cat. no. C0481; Sigma-Aldrich; Merck $\mathrm{KGaA}$ ). The concentration of protein was detected using BCA protein assay kit (Thermo Fisher Scientific, Inc.). A total of $30 \mu \mathrm{g}$ of protein was added to $50 \mu \mathrm{l}$ of Laemmli sample buffer (Sigma-Aldrich; Merck KGaA) and boiled for $5 \mathrm{~min}$. Proteins (30 $\mu \mathrm{g}$ per lane) were then separated on $10-20 \%$ SDS-PAGE and electrophoresed at $20 \mathrm{~V}$ for $3 \mathrm{~h}$. The 
Table I. Content of CRP, Il-6, IFN- $\gamma$ in serum of rats following septic shock model establishment.

\begin{tabular}{lccccc}
\hline Parameters & Control & HT group & HNT group & LT group & LNT group \\
\hline CRP $(\mathrm{ng} / \mathrm{ml})$ & $303.02 \pm 16.21$ & $604.47 \pm 15.09^{\mathrm{a}}$ & $604.33 \pm 19.47^{\mathrm{a}}$ & $466.54 \pm 19.81^{\mathrm{a}, \mathrm{b}}$ & $459.23 \pm 17.79^{\mathrm{a}, \mathrm{b}}$ \\
IL-6 $(\mathrm{pg} / \mathrm{ml})$ & $14.64 \pm 2.83$ & $61.86 \pm 2.48^{\mathrm{a}}$ & $61.67 \pm 2.92^{\mathrm{a}}$ & $46.31 \pm 2.58^{\mathrm{a}, \mathrm{b}}$ & $45.73 \pm 2.73^{\mathrm{a}, \mathrm{b}}$ \\
IFN- $\gamma(\mathrm{pg} / \mathrm{ml})$ & $56.28 \pm 4.90$ & $349.75 \pm 8.56^{\mathrm{a}}$ & $354.52 \pm 9.65^{\mathrm{a}}$ & $127.53 \pm 7.05^{\mathrm{a}, \mathrm{b}}$ & $128.51 \pm 7.58^{\mathrm{a}, \mathrm{b}}$ \\
\hline
\end{tabular}

${ }^{\mathrm{a}} \mathrm{P}<0.01$ vs. the control group; ${ }^{\mathrm{b}} \mathrm{P}<0.05$ vs. the HT group. CRP, C-reactive protein; IL, interleukin; IFN, interferon; HT, high-inflammation treatment; HNT, high-inflammation non-treatment; LT, low-inflammation treatment; LNT, low-inflammation non-treatment.

Table II. Changes of the rats arterial pressure, heart rate, ALT and AST.

\begin{tabular}{lccrrr}
\hline Parameters & Control & HT group & HNT group & LT group & LNT group \\
\hline Arterial pressure & $102.01 \pm 5.15$ & $56.86 \pm 6.94^{\mathrm{a}}$ & $58.46 \pm 6.38^{\mathrm{a}}$ & $55.73 \pm 7.07^{\mathrm{a}}$ & $52.79 \pm 7.17^{\mathrm{a}}$ \\
Heart rate & $314.58 \pm 11.16$ & $496.17 \pm 13.11^{\mathrm{a}}$ & $496.42 \pm 13.45^{\mathrm{a}}$ & $491.92 \pm 10.75^{\mathrm{a}}$ & $493.42 \pm 11.24^{\mathrm{a}}$ \\
ALT (U/l) & $41.66 \pm 2.87$ & $94.15 \pm 5.47^{\mathrm{a}}$ & $95.20 \pm 5.32^{\mathrm{a}}$ & $81.19 \pm 1.98^{\mathrm{a}}$ & $82.68 \pm 3.53^{\mathrm{a}}$ \\
AST (U/l) & $116.30 \pm 4.96$ & $181.77 \pm 6.33^{\mathrm{a}}$ & $184.55 \pm 7.09^{\mathrm{a}}$ & $165.72 \pm 6.15^{\mathrm{a}}$ & $167.15 \pm 4.53^{\mathrm{a}}$
\end{tabular}

${ }^{\text {aP }}<0.01$ vs. the control group. ALT, alanine aminotransferase; AST, aspartate aminotransferase; HT, high-inflammation treatment; HNT, high-inflammation non-treatment; LT, low-inflammation treatment; LNT, low-inflammation non-treatment.

proteins were separated according to their molecular weight. Protein samples were transferred to polyvinylidene difluoride membranes (EMD Millipore), which were subsequently blocked with $5 \%$ skimmed milk at $4^{\circ} \mathrm{C}$ overnight. Membranes were incubated with primary antibodies anti-phosphorylated (p)-p38 mitogen-activated protein kinase (MAPK; 1:1,000; cat. no. ab195049; Abcam), anti-p38MAPK (1:1,000; cat. no. ab31828; Abcam), anti-p-NF-кB-p65 (1:2,000; cat. no. ab86299; Abcam) and anti-NF-кB-p65 (1:1,000; cat. no. ab16502; Abcam) overnight at $4^{\circ} \mathrm{C}$. Membranes were washed with PBS three times then incubated with horseradish peroxidase-labeled goat anti-rabbit IgG (1:2,000; cat. no. ab6721; Abcam) at $37^{\circ} \mathrm{C}$ for $1 \mathrm{~h}$. Membranes were washed with PBS three times and protein band were visualized using enhanced chemiluminescence detection system (Thermo Fisher Scientific, Inc.). Gray-scale scanning and quantification were performed using Image J software (version 1.32; National Institutes of Health) with protein levels normalized to $\beta$-actin.

Statistical analysis. Data were analyzed using SPSS19.0 statistical software (SPSS, Inc.). Data were expressed as the mean \pm standard deviation. Comparisons between two groups were analyzed using Student's t-test. One-way analysis of variance was used for data analysis between multiple groups with a Least Significant Difference post-hoc test. $\mathrm{P}<0.05$ was considered to indicate statistical significance.

\section{Results}

Rat serum inflammatory factor content increases during inflammation. According to the levels of CRP, IL-6 and IFN- $\gamma$ in the model groups, 48 rats were divided into a high-inflammation treatment group (HT group), a high-inflammation non-treatment group (HNT group), a low-inflammation treatment group (LT group) and a low-inflammation non-treatment group (LNT group). Rats were classed as high-inflammation when levels of CRP $\geq 500 \mathrm{ng} / \mathrm{ml}$, IL- $6 \geq 50 \mathrm{pg} / \mathrm{ml}$ and IFN- $\gamma \geq 150 \mathrm{pg} / \mathrm{ml}$. CRP, IL-6 and IFN- $\gamma$ levels in all model groups were significantly increased compared with the control group $(\mathrm{P}<0.01$; Table I). The serum contents of CRP, IL- 6 and IFN- $\gamma$ in the low inflammation group were significantly lower than those of the high inflammation group $(\mathrm{P}<0.05$; Table I).

Liver damage indicators increase during inflammation. The arterial pressures of all model groups were significantly lower compared with the control group $(\mathrm{P}<0.01$; Table II). Similarly, heart rate, ALT and AST levels were significantly higher compared with the control group $(\mathrm{P}<0.01$; Table II). However, there was no significant difference amongst inflammation groups of the model group ( $\mathrm{P}>0.05$; Table II).

Serum CRP, IL-6 and IFN- $\gamma$ levels decrease in high-inflammation septic shock rats following glucocorticoid therapy. There were no marked differences in serum CRP, IL-6 and IFN- $\gamma$ levels between the HT group and control group following 7 days of treatment ( $P>0.05$; Fig. 1). Before 7 days of treatment, the CRP, IL- 6 and IFN- $\gamma$ levels in the four model groups were significantly higher than control group. In the LT group, following 7 days of treatment, the content of serum CRP, IL- 6 and IFN- $\gamma$ was significantly higher compared with the control group $(\mathrm{P}<0.01$; Fig. 1$)$. The content of CRP, IL- 6 and IFN- $\gamma$ in HNT group at each time point was significantly higher compared with the control and HT groups $(\mathrm{P}<0.01$; Fig. 1). However, no marked differences were identified between the LNT group and LT group $(\mathrm{P}>0.05)$. These results demonstrated that steroid glucocorticoid therapy was more effective in attenuating 

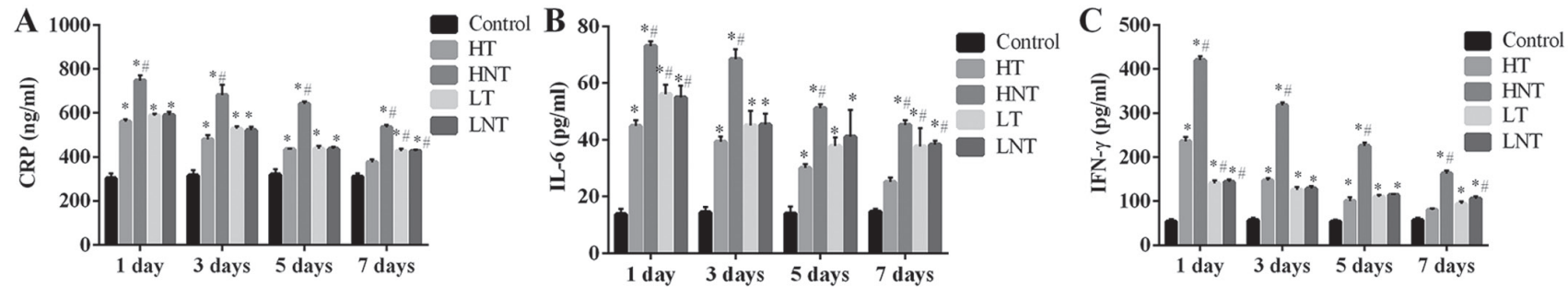

Figure 1. Serum levels of CRP, IL-6 and IFN- $\gamma$ are decreased in high-inflammation septic shock model rats following glucocorticoid therapy. (A) CRP, (B) IL-6 and (C) IFN- $\gamma$ levels were measured on days 1, 3, 5 and 7 following the establishment of a septic shock model $(\mathrm{n}=12) .{ }^{*} \mathrm{P}<0.01$ vs. the control group; ${ }^{*} \mathrm{P}<0.01$ vs. the HT group. CRP, C-reactive protein; IL, interleukin; IFN, interferon; HT, high-inflammation treatment; HNT, high-inflammation non-treatment; LT, low-inflammation treatment; LNT, low-inflammation non-treatment.
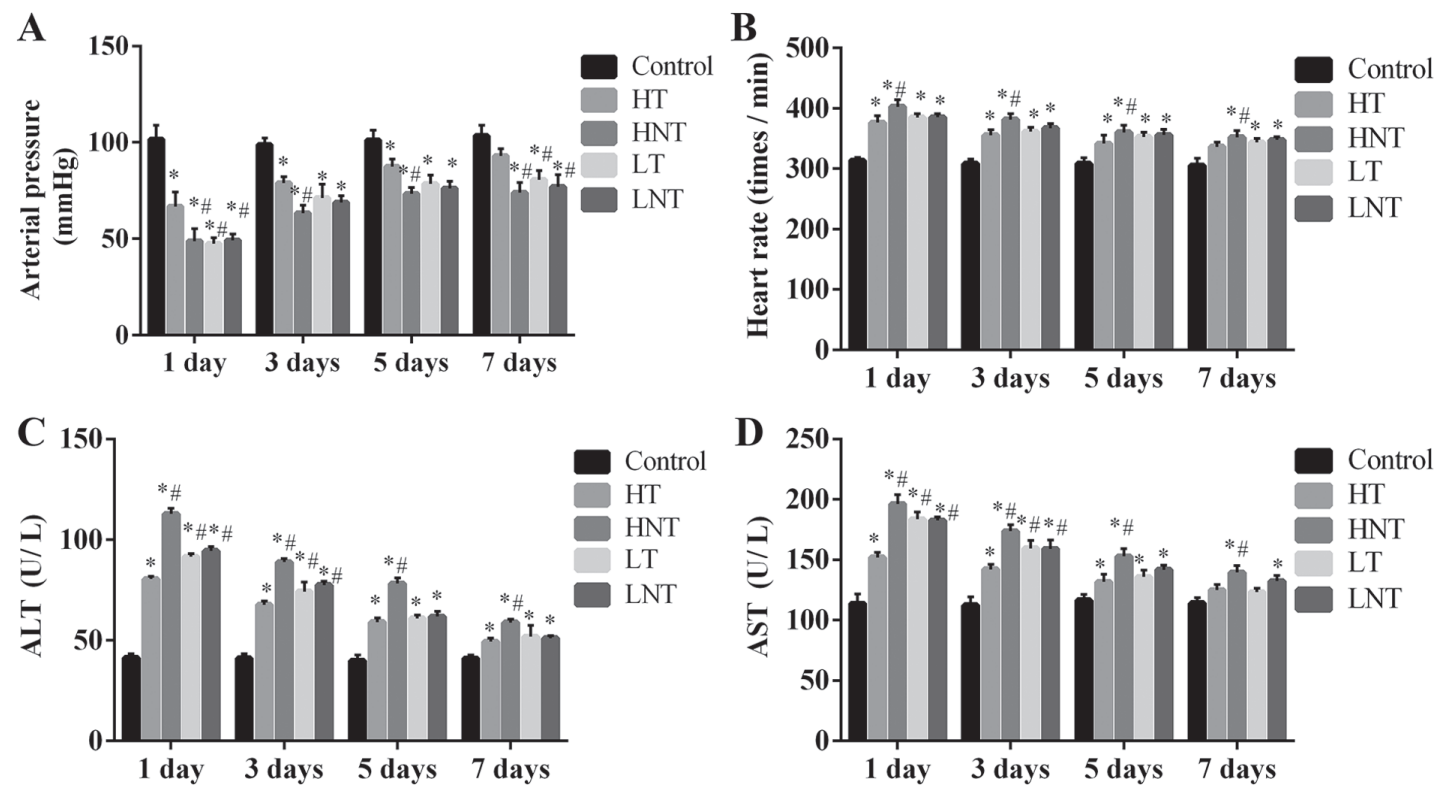

Figure 2. Arterial pressure and liver function increases and heart rate decreases following glucocorticoid therapy of high-inflammation septic shock model rats. (A) Arterial pressure, (B) heart rate, (C) content of ALT and (D) content of AST over 7 days in high and low inflammation rats, with and without glucocorticoid treatment ( $\mathrm{n}=12$ ). ${ }^{\mathrm{P}} \mathrm{P}<0.01$ vs. control group; ${ }^{~} \mathrm{P}<0.01$ vs. HT group. ALT, alanine aminotransferase; AST, aspartate aminotransferase; HT, high-inflammation treatment; HNT, high-inflammation non-treatment; LT, low-inflammation treatment; LNT, low-inflammation non-treatment.

inflammation in the high-inflammation group compared with the low-inflammation group.

Arterial pressure and liver function increases and heart rate decreases following the glucocorticoid therapy of high-inflammation septic shock model rats. Following 7 days of treatment, the arterial pressure of HT rats was significantly increased compared with the other model groups $(\mathrm{P}<0.01$; Fig. 2A), but revealed no significant difference compared with the control group $(\mathrm{P}>0.05$; Fig. $2 \mathrm{~A})$. The arterial pressure of rats in LT group was also significantly lower than those of the HT group ( $\mathrm{P}<0.01$; Fig. 2A). Heart rate decreased for HT and LT group after treatment and there were no marked differences for HT rats compared with the control group following 7 days of treatment ( $\mathrm{P}>0.05$; Fig. 2B). At day 1, levels of serum ALT and AST in each model group were significantly increased compared with the control group. Furthermore, the content of ALT and AST in the LT group was significantly increased compared with the HT group at day $1(\mathrm{P}<0.01$; Fig. $2 \mathrm{C}$ and D). Following 7 days of treatment, in comparison with control group, serum ALT in HT group was significantly higher $(\mathrm{P}<0.01)$, whilst AST levels was no significant difference $(\mathrm{P}>0.05)$. ALT was slightly higher in the LT group compared with the HT group, but not significantly ( $\mathrm{P}>0.05$; Fig. 2C and D).

Pathological changes of septic shock model rats. Fig. 3 demonstrates that the liver tissue structure of the control rats was intact and clear, without edema or inflammation. In the HT group, the hepatic sinusoids were dilated with local congestion. At day 3, there was hepatic sinusoidal dilatation and portal area fibrosis. At day 5, hepatic lobular hepatic sinusoidal micro dilatation and microthrombus formation was observed. Hepatocytes were uniform in size and comparable to normal tissues on 7 days. In the HNT group, hepatic sinusoids expanded with necrosis at day 1. Hepatic sinusoidal expansion, necrosis, hepatocytes size were heterogeneous at day 3. On 5 days there were areas of necrosis and collapse of hepatic lobular structure, inflammatory cell infiltration. Following 7 days, there were areas of necrosis and fibrosis in portal area and hepatic sinusoidal dilation. In the LT group, hepatic sinusoids extended, hepatocytes were dead and portal area enlarged at day 1 . On day 3 , the structure of hepatic 
1 day
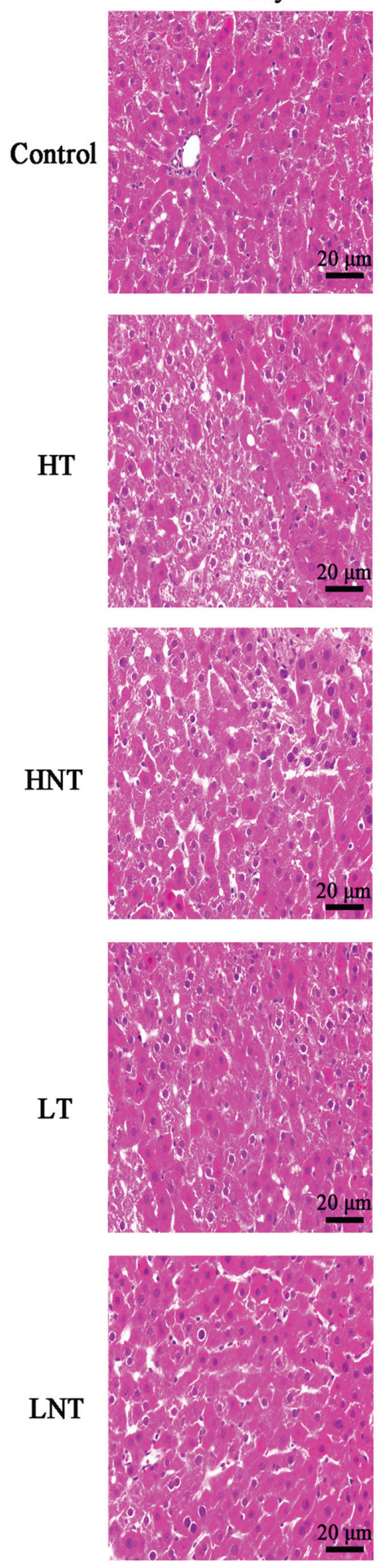

3 days
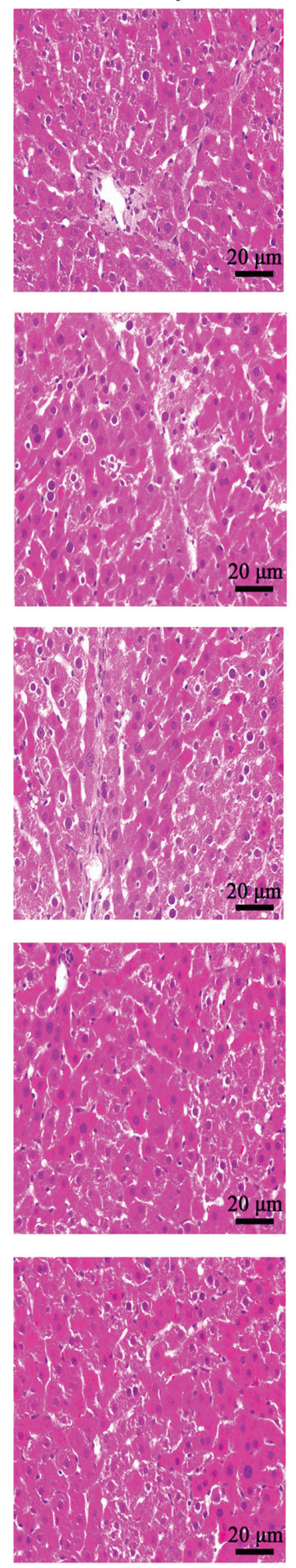

5 days
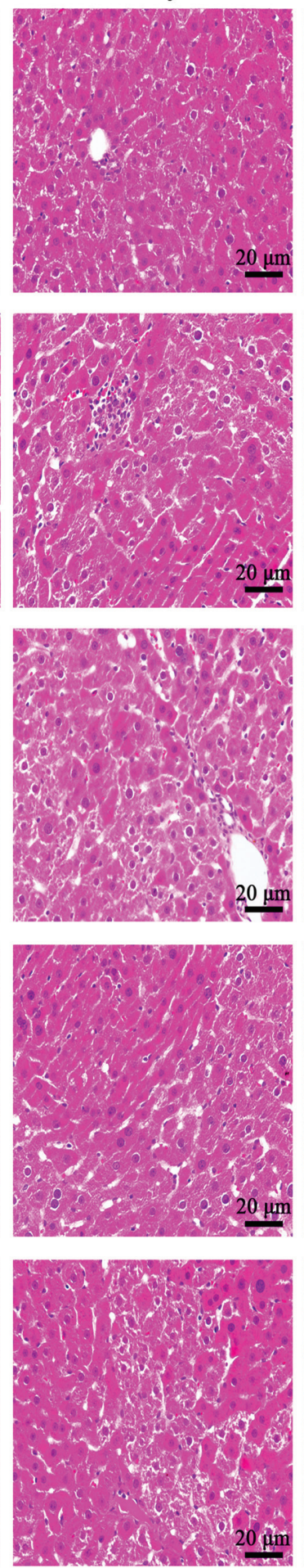

7 days
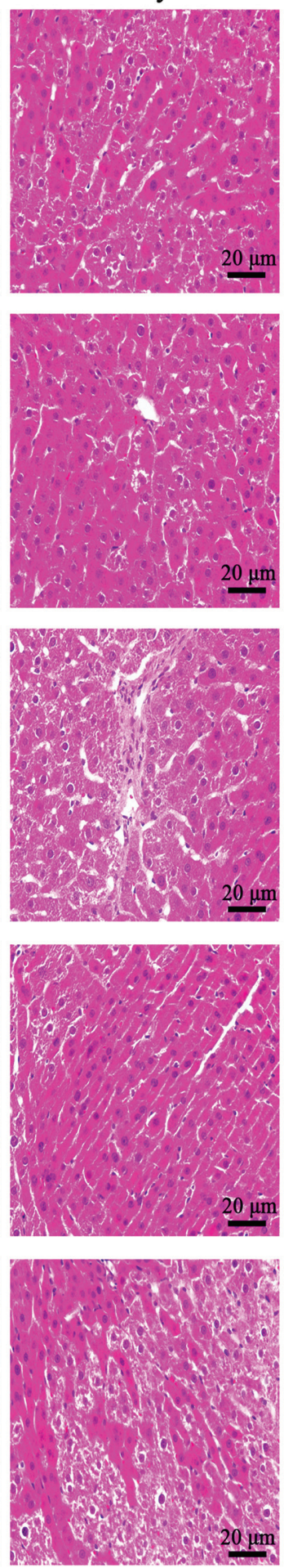

Figure 3. Hematoxylin and eosin staining observing the pathological changes of the liver over 7 days in high and low inflammation rats, with and without glucocorticoid treatment (magnification, x400; scale bar, $20 \mu \mathrm{m}$ ). HT, high-inflammation treatment; HNT, high-inflammation non-treatment; LT, low-inflammation treatment; LNT, low-inflammation non-treatment.

lobule was abnormal and there was hepatic sinusoidal dilation and necrosis. At day 5, hepatic lobular structure disappeared, hepatic sinusoids expanded and necrosis was apparent. Hepatic lobule structure was abnormal, hepatic sinusoidal dilatation occurred, portal area was enlarged and necrosis was present at 7 days. In LNT group, hepatic sinusoidal dilatation occurred, portal area was enlarged, hepatic cell underwent vacuolization at day 1 . Hepatic sinusoids dilated, hepatocyte swelled, necrosis occurred and microthrombus formed at day 3. Hepatic sinusoidal dilation was present, hepatic cord disappeared and microthrombus formation occurred at day 5. Hepatic lobule structure had almost disappeared, hepatic sinusoid expanded and sheet necrosis was observed at day 7 . 
A

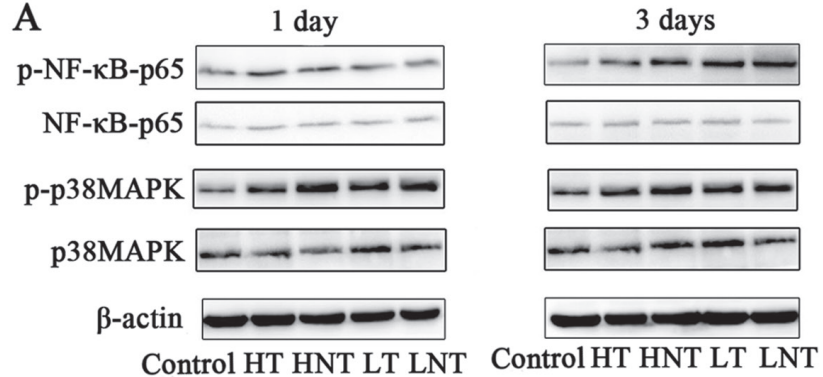

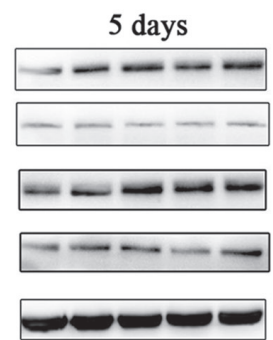

Control HT HNT LT LNT Control HT HNT LT LNT
B

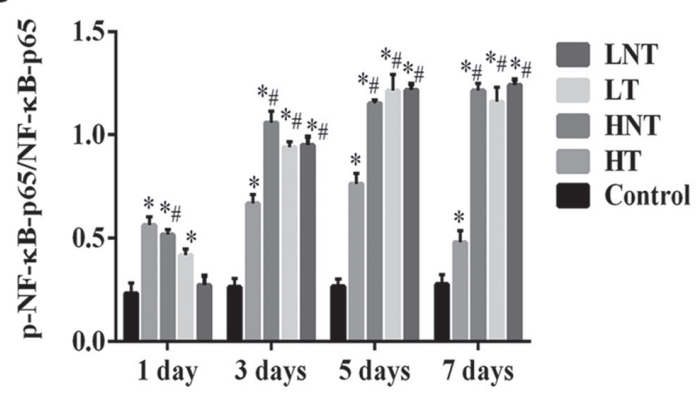

$\mathrm{C}$

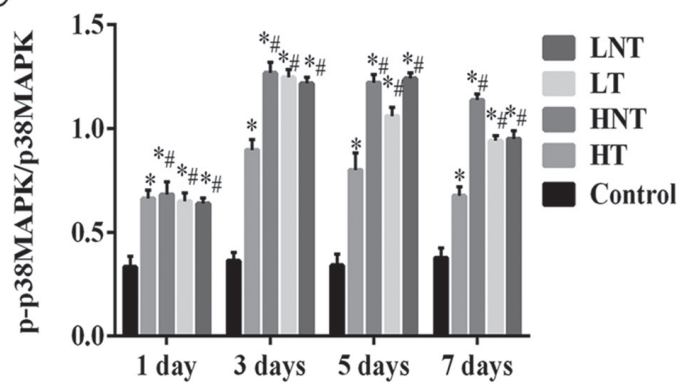

Figure 4. Phosphorylation of NF-кB-p65 and p38MAPK decreases following glucocorticoid treatment of high-inflammation septic shock model rats. (A) Representative western blot analysis and (B) quantification of the p-NFkB-p65 protein and (C) p-p38MAPK protein over 7 days in high and low inflammation rats, with and without glucocorticoid treatment. ${ }^{*} \mathrm{P}<0.01$ vs. control group; ${ }^{*} \mathrm{P}<0.01$ vs. HT group. p, phosphorylation; MAPK, mitogen-activated protein kinase; HT, high-inflammation treatment; HNT, high-inflammation non-treatment; LT, low-inflammation treatment; LNT, low-inflammation non-treatment.

Phosphorylation of NF- $\kappa B-p 65$ and p38MAPK decreases following glucocorticoid treatment of high-inflammation septic shock model rats. From day 3 onwards, the phosphorylation levels of NF- $\kappa$ B-p65 (Fig. 4A and B) and p38MAPK (Fig. 4A and C) proteins in the HT group were significantly decreased compared with the other model groups $(\mathrm{P}<0.01)$. LT, LNT and HNT groups displayed significantly higher phosphorylation compared with the control group $(\mathrm{P}<0.01$; Fig. 4). The level of NF- $\kappa$ B-p65 protein phosphorylation was highest in LNT group, and the level of p38MAPK protein phosphorylation was highest in HNT group, whilst there was no obvious difference between LT group and LNT group at day 7. These results demonstrated that glucocorticoid treatment had more beneficial effect in the high-inflammation group compared with the low-inflammation group.

\section{Discussion}

Glucocorticoids are considered to be the most effective anti-inflammatory drugs (21), but their therapeutic role in sepsis remains controversial, particularly regarding the proper dose of them. A large number of clinical studies have determined that the concentration of inflammatory factors in low in the blood of sepsis patients receiving hydrocortisone $(22,23)$. Furthermore, glucocorticoids are immunosuppressive agents, and different degrees of immune function inhibition may occur in the course of application. A previous study demonstrated that high doses of glucocorticoids increases the possibility of double infection and mortality, therefore the use of high doses of glucocorticoids was prohibited (24). Hydrocortisone treatment significantly attenuates proinflammatory cytokines in patients (25). The early application of low-dose hydrocortisone can improve patient prognosis (26).
The present study grouped rats according to the level of blood inflammatory factors to evaluate the effectiveness of hydrocortisone in the treatment of high-inflammation and low-inflammation. The results demonstrated that CRP, IL-6 and IFN- $\gamma$ HT rat serum levels decreased significantly following 7 days of glucocorticoid treatment, with the arterial pressure, heart rate and liver function of rats ameliorated compared with the low-inflammation treatment group. There was no obvious difference between HT treatment compared with the control group following 7 days of treatment. The results of $H \& E$ staining determined that following 7 days of treatment with hydrocortisone in the HT, rat liver tissue structure was recovered significantly compared with rats in the LT group. Taken together, these results demonstrated that the treatment effect of hydrocortisone on the high-inflammation group was superior to the low-inflammatory group.

Current research on glucocorticoid treatment for patients with severe sepsis recommends early treatment (27); however, there is no clear standard for 'early'. The early clinical manifestations of patients with severe sepsis and the corresponding monitoring of immune inflammatory factors in the blood are factors used to determine the use of glucocorticoid therapy. Domestic and international studies on the timing of glucocorticoid use in patients with severe sepsis are rare. Therefore, the timing of glucocorticoid adjuvant therapy for patients with septic shock is currently inconclusive. Therefore, the present study based its results on the level of inflammatory factors.

The p38 MAPK signaling pathway is crucial in cell signal transduction, disease development and the progression of sepsis. It is one of the key pathways leading to the occurrence of multiple organ dysfunction (28). NF- $\mathrm{B}$ serves a central role in the development of inflammatory reactions. The activation and overexpression of NF- $\kappa \mathrm{B}$ causes an imbalance of cytokines and 
produces a cascade effect of inflammatory responses $(29,30)$. Studies have also demonstrated that the p38MAPK/NF- $\mathrm{B}$ signaling pathway is activated in the course of various diseases, including sepsis and certain inflammatory reactions $(31,32)$. Additionally, its excessive activation is closely associated with the development and severity of the disease. The present study examined the expression of p38MAPK and NF- $\mathrm{B}-\mathrm{p} 65$ in different inflammation groups and evaluated the effect of glucocorticoid treatment on the $\mathrm{p} 38 \mathrm{MAPK} / \mathrm{NF}-\kappa \mathrm{B}$ signaling pathway. The results determined that the phosphorylation levels of p38MAPK protein and NF- $\mathrm{B}-\mathrm{p} 65$ protein in all the model groups were significantly increased compared with the control. Following treatment with hydrocortisone, the protein expression in the HT group decreased significantly, and the phosphorylation level was significantly inhibited after 7 days of treatment. The present results determined that glucocorticoids have a better protective effect on the liver of septic shock rats with high-inflammation compared with low inflammation. The present study hypothesized that this effect may be due to the strong anti-inflammatory effects of glucocorticoids quickly inhibiting high-level inflammation. A limitation of the present study is that the pathological changes were only observed in the liver. Future work will investigate whether glucocorticoids exert the same protective effects on lung and kidney tissue in high inflammatory septic rats and will elucidate the underlying reaction mechanism.

In conclusion, the present study analyzed the effects of glucocorticoids on inflammatory markers, liver function and tissue changes in septic shock rats. The findings provided a theoretical basis for the use of glucocorticoids in the treatment of septic shock according to the level of inflammation.

\section{Acknowledgements}

Not applicable.

\section{Funding}

No funding was received.

\section{Availability of data and materials}

The datasets used and/or analyzed during the current study are available from the corresponding author on reasonable request.

\section{Authors' contributions}

XL, ML and YL designed the study. XL, ML, LL, XT and YL performed experiments. XL, ML, LL and XT performed data analysis, interpreted the data and acquired samples. XL and ML contributed to pathological analysis. XL, ML and YL wrote the manuscript. All authors read and approved the final manuscript.

\section{Ethics approval and consent to participate}

The present study was approved by Ethics Committee of the Affiliated Yantai Yuhuangding Hospital of Qingdao University.

\section{Patient consent for publication}

Not applicable.

\section{Competing interests}

The authors declare that they have no competing interests.

\section{References}

1. Schulte W, Bernhagen J and Bucala R: Cytokines in Sepsis: Potent immunoregulators and potential therapeutic targets-an updated view. Mediators Inflamm 2013: 165974, 2013.

2. Yan J, Li S and Li S: The role of the liver in sepsis. Int Rev Immunol 33: 498-510, 2014.

3. Ryan T, Coakley JD and Martinloeches I: Defects in innate and adaptive immunity in patients with sepsis and health care associated infection. Ann Transl Med 5: 447, 2017.

4. Dewitte A, Lepreux S, Villeneuve J, Rigothier C, Combe C, Ouattara A and Ripoche J: Blood platelets and sepsis pathophysiology: A new therapeutic prospect in critical [corrected] ill patients? Ann Intensive Care 7: 115, 2017.

5. Shi LL and Han YQ: Research advance of sepsis related coagulation and inflammation biomarkers. Medical Recapitulate 5: 873-877, 2016 (In Chinese).

6. Spoto S, Valeriani E and Costantino S: Nosography of systemic inflammatory response syndrome, sepsis, severe sepsis, septic shock and multiple organ dysfunction syndrome in internal medicine patients. Italian J Med 9: 243-251, 2015.

7. Michalek J, Svetlikova P, Fedora M, Klimovic M, Klapacova L, Bartosova D, Hrstkova $\mathrm{H}$ and Hubacek JA: Interleukin-6 gene variants and the risk of sepsis development in children. Hum Immunol 68: 756-760, 2007.

8. Bozza FA, Salluh JI, Japiassu AM, Soares M, Assis EF, Gomes RN, Bozza MT, Castro-Faria-Neto HC and Bozza PT: Cytokine profiles as markers of disease severity in sepsis: A multiplex analysis. Crit Care 11: R49, 2007.

9. Alexandrov PN, Kruck TP and Lukiw WJ: Nanomolar aluminum induces expression of the inflammatory systemic biomarker $\mathrm{C}$-reactive protein $(\mathrm{CRP})$ in human brain microvessel endothelial cells (hBMECs). J Inorg Biochem 152: 210-213, 2015.

10. Mayer CM, Gruben HJ, Land EM, Scharnag H and Marz W: W12-P-038 Statin induced inhibition of CRP expression in primary human hepatocytes. Atherosclerosis 6: 70-71, 2005.

11. Memiş D, Gursoy O, Tasdogan M, Süt N, Kurt I, Türe M and Karamanlioğlu B: High C-reactive protein and low cholesterol levels are prognostic markers of survival in severe sepsis. J Clin Anesth 19: 186-191, 2007.

12. Ji Y, Zheng MF, Ye SG, Wu XB and Chen JY: Agrocybe aegerita polysaccharide combined with chemotherapy improves tumor necrosis factor- $\alpha$ and interferon- $\gamma$ levels in rat esophageal carcinoma. Dis Esophagus 26: 859-863, 2013.

13. Valentine L, Potts R and Premenko-Lanier M: CD8+ T cell derived IFN- $\gamma$ prevents infection by a second heterologous virus. J Immunol 189: 5841-5848, 2012.

14. Lin CF, Lin CM, Lee KY, Wu SY, Feng PH, Chen KY, Chuang HC, Chen CL, Wang YC, Tseng PC and Tsai TT: Escape from IFN- $\gamma$-dependent immunosurveillance in tumorigenesis. J Biomed Sci 24: 10, 2017.

15. Conway-Campbell BL, George CL, Pooley JR, Knight DM, Norman MR, Hager GL and Lightman SL: The HSP90 molecular chaperone cycle regulates cyclical transcriptional dynamics of the glucocorticoid receptor and its coregulatory molecules $\mathrm{CBP} / \mathrm{p} 300$ during ultradian ligand treatment. Mol Endocrinol 25: 944-954, 2011.

16. Oppert M, Schindler R, Husung C, Offermann K, Gräf KJ, Boenisch O, Barckow D, Frei U and Eckardt KU: Low-dose hydrocortisone improves shock reversal and reduces cytokine levels in early hyperdy-namic septic shock. Crit Care Med 33: 2457-2464, 2005.

17. Xiang G, Fan M, Ma Y, Wang M, Gao J, Chen J, Li X, Xue W, Wang Y, Gao H, et al: Anti-inflammatory actions of Caesalpinin M2 in experimental colitis as a selective glucocoricoid receptor modulator. Biochem Pharmacol 150: 150-159, 2018. 
18. Annane D, Bellissant E, Bollaert PE, Briegel J, Confalonieri M De Gaudio R, Keh D, Kupfer Y, Oppert M and Meduri GU: Corticosteroids in thetreatment of severe sepsis and septic shock in adults: A systematic review. JAMA 301: 2362-2375, 2009.

19. Laviolle B, Annane D, Fougerou C and Bellissant E: Gluco- and mineralocorticoid biological effects of a 7-day treatment with low doses of hydrocortisone and fludrocortisone in septic shock. Intensive Care Med 38: 1306-1314, 2012.

20. Shapiro NI, Trzeciak S, Hollander JE, Birkhahn R, Otero R, Osborn TM, Moretti E, Nguyen HB, Gunnerson K, Milzman D, et al: The diagnostic accuracy of plasma neutrophil gelatinase-associated lipocalin in the prediction of acute kidney injury in emergency department patients with suspected sepsis. Ann Emerg Med 56: 52-59.e1, 2010.

21. Cheng B, Xie G, Yao S, Wu X, Guo Q, Gu M, Fang Q, Xu Q, Wang D, Jin Y, et al: Epidemiology of severe sepsis in critically ill surgical patients in ten university hospitals in China. Crit Care Med 35: 2538-2546, 2007.

22. Yu H, Chi D, Wang S and Liu B: Effect of early goal-directed therapy on mortality in patients with severe sepsis or septic shock: A meta-analysis of randomised controlled trials. BMJ Open 6: e008330, 2016.

23. Walton E, Oliveros H and Villamor E: Hemoglobin concentration and parasitemia on hospital admission predict risk of multiple organ dysfunction syndrome among adults with malaria. Am J Trop Med Hyg 91: 50-53, 2014.

24. Bone R, Fisher CJ Jr, Clemmer TP, Slotman GJ, Metz CA and Balk RA: A controlled trial of high-dose methylprednisolone in the treatment of severe sepsis and septic shock. N Engl J Med 317: 653-658, 1987.
25. Zhao Y and Ding C: Effects of hydrocortisone on regulating inflammation, hemodynamic stability, and preventing shock in severe sepsis patients. Med Sci Monit 24: 3612-3619, 2018.

26. Greenberg SB and Coursin DB: Timing of corticosteroids in refractory septic shock: A key or wishful thinking? Crit Care Med 42: 1733-1735, 2014.

27. Lu J, Wang X, Chen Q, Chen M, Cheng L, Dai L, Jiang H and Sun Z: The effect of early goal-directed therapy on mortality in patients with severe sepsis and septic shock: A meta-analysis. J Surg Res 202: 389-397, 2016.

28. Yousif NG, Hadi NR, Alamran F and Zigam QA: Cardioprotective effects of irbesartan in polymicrobial sepsis: The role of the p38MAPK/NF- $\mathrm{B}$ signaling pathway. Herz 43: 140-145, 2018.

29. Cuzzocrea S, Crisafulli C, Mazzon E, Esposito E, Muià C, Abdelrahman M, Di Paola R and Thiemermann C: Inhibition of glycogen synthase kinase-3beta attenuates the development of carrageenan-induced lung injury in mice. Br J Pharmacol 149: 687-702, 2006

30. Eisenhut M: Comment on 'Duration and intensity of NF-kappa B activity determine the severity of endotoxin-induced acute lung injury'. J Immunol 177: 2038, 2006.

31. Kim SH and Shin TY: Anti-inflammatory effect of leaves of Eriobotrya japonica, correlating with attenuation of p38 MAPK, ERK and NF-kappaB activation in mast cells. Toxicol In Vitro 23: 1215-1219, 2009.

32. Ishibashi Y, Matsui T, Ueda S, Fukami K and Yamagishi SI: Advanced glycation end products potentiate citrated plasma-evoked oxidative and inflammatory reactions in endothelial cells by up-regulating protease-activated receptor-1 expression. Cardiovasc Diabetol 13: 60, 2014. 